\title{
Clinical Audit on the Use of Continuous Positive Airway Pressure in Neonatal Intensive Care Unit of Assiut University Children Hospital
}

\author{
CATREEN F. MOUSA, M.Sc.; AZZA A. EL-TAYEB, M.D. and AMIRA M. AHMED, M.D.
}

The Department of Pediatrics, Faculty of Medicine, Assiut University, Assiut, Egypt

\begin{abstract}
Background: Continuous Positive Airway Pressure (CPAP) is a well-established mode of respiratory support in preterm newborns. We aim by this study to assess adherence of medical staff to the guidelines and adapted protocols of using CPAP.

Aim of Study: To assess the application of medical staff for the guidelines and adapted protocols of using CPAP in Neonatal Intensive Care Unit at Assuit University Children Hospital.

Patients and Methods: This study is a prospective observational analysis of hospital admission of neonates receiving CPAP care in the Neonatal Intensive Care Unit in Assuit University Children Hospital.

Results: Outcome of CPAP use was good as improvement of cases occurred in $58.3 \%$ of cases and failed in $41.7 \%$ of cases.

Conclusion: Data of the study showed that using CPAP for cases of respiratory distress in neonatal Intensive Care Unit in Assiut University Children Hospital partially followed the reference standard of the study.
\end{abstract}

Key Words: Continuous positive airway pressure - Neonatal respiratory distress - Newborn - Respiratory distress syndrome - Mechanical ventilation.

\section{Introduction}

CONTINUOUS Positive Airway Pressure (CPAP) is a well-established mode of respiratory support in preterm newborns. Advancement in technology, increasing survival of extremely preterm newborns and better understanding of various respiratory diseases led to new evidence in this field over last decade. It is important to update ourselves on the recent changes in the practice of CPAP and its implications for resource-limited settings [1]

Current modalities of ventilatory assistance in the management of respiratory distress in infants range from Continuous Positive Airway Pressure

Correspondence to: Dr. Catreen F. Mousa,

E-Mail: littleangle2017@yahoo.com
(CPAP) to various modes of mechanical ventilation. The use of CPAP has been associated with a lower incidence of chronic lung disease when used as the initial respiratory support. Also, mechanical ventilation has the potential to injure the airways and lung parenchyma [2]

Recently, there has been a renewed interest in gentle ventilation strategies, such as CPAP as a way to improve outcomes for very preterm infants. A wide variety of devices are used to deliver CPAP, including single or binasal prongs where pressure is generated by a column of water (bubble CPAP) or a ventilator. Bubble CPAP (BCPAP) is appealing because of its simplicity and low cost [2].

\section{Patients and Methods}

This study aimed to evaluate how much the adopted protocols of using CPAP are applied in neonatal Intensive Care Unit in Assiut University Children Hospital from 1 st of June 2015 to 30 th of January 2017. The adopted protocols used followed the American association respiratory care guidelines 2004 and Queensland clinical guidelines 2014 which is locally approved by the Neonatal Intensive Care Unit in Assiut University Children Hospital. Data collection was done by reviewing the sheets and direct observation of patients receiving CPAP care in NICU of AUCH during the study duration. The study included 60 neonates with respiratory distress receiving BCPAP care. Their ages ranged from $27 w k s$ to $40 w k s$ gestational age and post natal age from 1 to 25 days old ( 27 cases were males and 33 were females).

\section{Inclusion criteria:}

Neonates less than or equal 28 days old receiving CPAP care due to various causes of respiratory distress. 


\section{Exclusion criteria:}

Neonates more than or equal 28 days old.

Neonates with respiratory distress receiving other methods of respiratory support rather than CPAP.

An observational checklist based on the American association respiratory care guidelines 2004 and Queensland clinical guidelines 2014 was developed by investigators in order to asses adherence of medical staff to the guidelines and adapted protocols of using NCPAP.

\section{Parameters assessed:}

- Complete history taking which included gestational age (wks), mode of delivery, occurrence of obstructed labor, history of fetal hypoxia, administration of surfactant, history of $\mathrm{O}_{2}$ administration, mode of $\mathrm{O}_{2}$ administration \& percentage of $\mathrm{O}_{2}$ used, performance of abdominal or chest surgery and weaning from mechanical ventilation.

- Complete examination which included gestational age, weight, vital signs (temp., pulse, respiratory rate, blood pressure), umbilical stump \& nails condition, abdominal, cardiac and chest examination.

- Investigations which included blood gases analysis, ECG monitoring, chest X-ray, pulse oximeter measurements.

- Indications of connection according to resident doctors (provisional diagnosis).

- Evaluation of CPAP device which included blended air/oxygen supply, flow meter at 5-10 1/m, humidifier water level, whether excess rainout in the afferent tubing was drained, nasal prong size, nasal prongs position or nasal mask, hat fits, corrugated tubing placement, whether head position was correct, tape at $7 \mathrm{~cm}$ at base of bottle, gas bubbling.

- Monitoring of the baby on nasal CPAP the following systems were checked every 2-4 hours which included respiratory (respiratory rate, grunting, nasal flaring, breath sounds), temperature, cardiovascular (blood pressure, auscultation) neurological (tone, activity), gastro-intestinal (free passage of stool, abdominal distension, intestinal sounds.

- Follow-up investigations which included blood gases analysis, ECG monitoring, chest X-ray, pulse oximeter measurements.
- Weaning of CPAP according to Queensland clinical guidelines 2014.

- Outcome of CPAP used.

\section{Results}

Table (1): Recorded demographic data.

\begin{tabular}{lll}
\hline & No. & $\%$ \\
\hline Gender: & & \\
$\quad$ Male & 27 & 45.0 \\
$\quad$ Female & 33 & 55.0 \\
Gestational age: & & \\
$\quad$ Range & $27-40 \mathrm{wks}$ & \\
Preterm (less than 37 weeks) & 52 & 86.7 \\
Full term (more than or equal 37 weeks) & 8 & 13.3 \\
\hline
\end{tabular}

Table (2): Recorded data about history of the cases.

\begin{tabular}{|c|c|c|c|c|}
\hline & \multicolumn{2}{|c|}{ Recorded } & \multicolumn{2}{|c|}{ Not recorded } \\
\hline & No. & $\%$ & No. & $\%$ \\
\hline Gestational age: & 60 & 100.0 & 0 & 0.0 \\
\hline Range & \multicolumn{2}{|c|}{$\begin{array}{l}27-40 w k s \\
\text { gestational age }\end{array}$} & & \\
\hline Mean \pm SD & \multicolumn{2}{|c|}{$33.73 \pm 4.29$} & & \\
\hline Mode of delivery: & 60 & 100.0 & 0 & 0.0 \\
\hline NVD & 29 & 48.3 & & \\
\hline $\mathrm{CS}$ & 31 & 51.7 & & \\
\hline Obstructed labor: & 59 & 98.3 & 1 & 1.7 \\
\hline Yes & 7 & 11.7 & & \\
\hline No & 52 & 86.7 & & \\
\hline History of fetal hypoxia: & 59 & 98.3 & 1 & 1.7 \\
\hline Yes & 8 & 13.3 & & \\
\hline No & 51 & 85.0 & & \\
\hline \multicolumn{5}{|l|}{ History of administration of } \\
\hline surfactant: & 60 & 100.0 & 0 & 0.0 \\
\hline Yes & 12 & 20.0 & & \\
\hline No & 48 & 80.0 & & \\
\hline History of $\mathrm{O} 2$ administration: & 60 & 100.0 & 0 & 0.0 \\
\hline Yes & 60 & 100.0 & & \\
\hline No & 0 & 0.0 & & \\
\hline $\begin{array}{l}\text { Mode of } \mathrm{O} 2 \text { administration \& } \\
\text { percentage of } \mathrm{O} 2 \text { used: }\end{array}$ & 59 & 98.3 & 1 & 1.7 \\
\hline Yes & 55 & 91.7 & & \\
\hline No & 4 & 6.7 & & \\
\hline $\begin{array}{l}\text { History of abdominal or chest } \\
\text { surgery: }\end{array}$ & 60 & 100.0 & 0 & 0.0 \\
\hline Yes & 0 & 0.0 & & \\
\hline No & 60 & 100.0 & & \\
\hline $\begin{array}{l}\text { History of weaning from } \\
\text { mechanical ventilation: }\end{array}$ & 60 & 100 & 0 & 0.0 \\
\hline Yes & 13 & 21.7 & & \\
\hline No & 47 & 78.3 & & \\
\hline
\end{tabular}


Table (3): Recorded data about examination in studied cases.

\begin{tabular}{|c|c|c|c|c|}
\hline \multirow{2}{*}{ Examination } & \multicolumn{2}{|c|}{ Recorded } & \multicolumn{2}{|c|}{ Not recorded } \\
\hline & No. & $\%$ & No. & $\%$ \\
\hline $\begin{array}{l}\text { Gestational age: } \\
\text { - Range (wks) } \\
\text { - Mean } \pm \text { SD }\end{array}$ & $\begin{array}{c}60 \\
27-40 \\
33.73\end{array}$ & 100.0 & 0 & 0.0 \\
\hline $\begin{array}{l}\text { Weight (grams): } \\
\cdot \text { Mean } \pm \text { SD }\end{array}$ & $\begin{array}{l}60 \\
1523 .\end{array}$ & $\begin{array}{r}100.0 \\
849.11\end{array}$ & 0 & 0.0 \\
\hline $\begin{array}{c}\text { Temp }(\text { rectal or core })(c) \text { : } \\
\quad \text { Normal }(36.5-37.5) \\
\quad \cdot \text { High }(>37.5) \\
\quad \text { Low }(<36.5)\end{array}$ & $\begin{array}{l}60 \\
57 \\
1 \\
2\end{array}$ & $\begin{array}{l}100.0 \\
95.0 \\
1.7 \\
3.3\end{array}$ & 0 & 0.0 \\
\hline $\begin{array}{l}\text { Pulse (resting) }(\mathrm{b} / \mathrm{m}): \\
\cdot \text { Normal }(120-160) \\
\cdot \text { High }(>170) \\
\text { - Low }(<100)\end{array}$ & $\begin{array}{l}60 \\
44 \\
15 \\
1\end{array}$ & $\begin{array}{l}100.0 \\
73.3 \\
25.0 \\
1.7\end{array}$ & 0 & 0.0 \\
\hline $\begin{array}{l}\text { Respiratory rate }(\mathrm{c} / \mathrm{m}) \text { : } \\
\text { - High }(>60) \\
\cdot \text { Low }(<40)\end{array}$ & $\begin{array}{l}60 \\
59 \\
1\end{array}$ & $\begin{array}{l}100.0 \\
98.3 \\
1.7\end{array}$ & 0 & 0.0 \\
\hline $\begin{array}{l}\text { Blood pressure (according to } \\
\text { age \& our NICU protocol): } \\
\text { - Normal } \\
\text { - Low }\end{array}$ & $\begin{array}{l}60 \\
51 \\
9\end{array}$ & $\begin{array}{l}100.0 \\
85.0 \\
15.0\end{array}$ & 0 & 0.0 \\
\hline $\begin{array}{l}\text { Umbilical stumb \& nails: } \\
\text { • Normal } \\
\text { • Abnormal (meconium } \\
\text { stained) }\end{array}$ & $\begin{array}{l}60 \\
54 \\
6\end{array}$ & $\begin{array}{l}100.0 \\
90.0 \\
10.0\end{array}$ & 0 & 0.0 \\
\hline $\begin{array}{c}\text { Abdominal exam: } \\
\text { - Normal }\end{array}$ & $\begin{array}{l}59 \\
59\end{array}$ & $\begin{array}{l}98.3 \\
98.3\end{array}$ & 1 & 1.7 \\
\hline $\begin{array}{l}\text { Cardiac exam: } \\
\text { - Normal } \\
\text { - Abnormal }\end{array}$ & $\begin{array}{l}60 \\
58 \\
2\end{array}$ & $\begin{array}{l}100.0 \\
96.7 \\
3.3\end{array}$ & 0 & 0.0 \\
\hline $\begin{array}{l}\text { Chest examination: } \\
\text { Retraction: } \\
\quad \cdot \text { Yes }\end{array}$ & $\begin{array}{l}60 \\
60 \\
60\end{array}$ & $\begin{array}{l}100.0 \\
100.0 \\
100.0\end{array}$ & 0 & 0.0 \\
\hline $\begin{array}{l}\text { Significant apnea: } \\
\cdot \text { Yes } \\
\cdot \text { No }\end{array}$ & $\begin{array}{l}59 \\
18 \\
41\end{array}$ & $\begin{array}{l}98.3 \\
30.0 \\
68.3\end{array}$ & 1 & 1.7 \\
\hline $\begin{array}{c}\text { Grunting: } \\
\cdot \text { Y Yes } \\
\cdot \text { No }\end{array}$ & $\begin{array}{c}60 \\
41 \\
19\end{array}$ & $\begin{array}{l}100.0 \\
68.3 \\
31.7\end{array}$ & 0 & 0.0 \\
\hline $\begin{array}{c}\text { Adventious sounds: } \\
\text { • Yes } \\
\cdot \text { No }\end{array}$ & $\begin{array}{l}60 \\
6 \\
54\end{array}$ & $\begin{array}{l}100.0 \\
10.0 \\
90.0\end{array}$ & 0 & 0.0 \\
\hline
\end{tabular}

Table (4): Recorded data about investigations (pre connection).

\begin{tabular}{|c|c|c|c|c|c|c|c|c|}
\hline & \multicolumn{2}{|c|}{ Recorded } & \multicolumn{2}{|c|}{$\begin{array}{c}\text { Not } \\
\text { recorded }\end{array}$} & \multicolumn{2}{|c|}{ Done } & \multicolumn{2}{|c|}{$\begin{array}{l}\text { Not } \\
\text { done }\end{array}$} \\
\hline & No. & $\%$ & No. & $\%$ & No. & $\%$ & No. & $\%$ \\
\hline - Blood gases & 32 & 53.3 & 28 & 45.7 & 32 & 53.3 & 28 & 45.7 \\
\hline - Chest X-ray & 0 & 0.0 & 60 & 100.0 & 58 & 96.7 & 2 & 3.3 \\
\hline - Pulse oximeter & 0 & 0.0 & 60 & 100.0 & 60 & 100.0 & 0 & 0.0 \\
\hline - Continuous ECG & 0 & 0.0 & 60 & 100.0 & 0 & 0.0 & 60 & 100.0 \\
\hline $\begin{array}{l}\text { - Indications of } \\
\text { connection } \\
\text { according to } \\
\text { resident doctors }\end{array}$ & 60 & 100 & 0 & 0 & 60 & 100 & 0 & 0 \\
\hline
\end{tabular}

Table (5): Monitoring of CPAP device during use.

\begin{tabular}{|c|c|c|c|c|c|c|c|c|}
\hline & \multicolumn{2}{|c|}{ Recorded } & \multicolumn{2}{|c|}{$\begin{array}{l}\text { Not } \\
\text { recorded }\end{array}$} & \multicolumn{2}{|c|}{ Done } & \multicolumn{2}{|c|}{$\begin{array}{l}\text { Not } \\
\text { done }\end{array}$} \\
\hline & No. & $\%$ & No. & $\%$ & No. & $\%$ & No. & $\%$ \\
\hline $\begin{array}{l}\text { - Blended air/oxygen } \\
\text { supply is } \\
\text { appropriate. }\end{array}$ & 0 & 0.0 & 60 & 100.0 & 60 & 100.0 & 0 & 0.0 \\
\hline $\begin{array}{l}\text { - Flow meter at 5- } \\
10 \mathrm{I} / \mathrm{m} \text {. }\end{array}$ & 0 & 0.0 & 60 & 100.0 & 60 & 100.0 & 0 & 0.0 \\
\hline $\begin{array}{l}\text { - Check humidifier } \\
\text { water level is } \\
\text { correct. }\end{array}$ & 0 & 0.0 & 60 & 100.0 & 60 & 100.0 & 0 & 0.0 \\
\hline $\begin{array}{l}\text { - Nasal prongs } \\
\text { positioned correctly } \\
\& \text { not touching the } \\
\text { septum or nasal } \\
\text { mask. }\end{array}$ & 0 & 0.0 & 60 & 100.0 & 60 & 100.0 & 0 & 0.0 \\
\hline $\begin{array}{l}\text { - Check head position } \\
\text { is correct. }\end{array}$ & 0 & 0.0 & 60 & 100.0 & 60 & 100.0 & 0 & 0.0 \\
\hline $\begin{array}{l}\text { Corrugated tubing } \\
\text { correctly placed. }\end{array}$ & 0 & 0.0 & 60 & 100.0 & 60 & 100.0 & 0 & 0.0 \\
\hline $\begin{array}{l}\text { - Gas bubbling } \\
\text { continuously. }\end{array}$ & 0 & 0.0 & 60 & 100.0 & 60 & 100.0 & 0 & 0.0 \\
\hline
\end{tabular}

Table (6): Recorded data about investigations for follow-up.

\begin{tabular}{lcccccccc}
\hline & \multicolumn{3}{c}{ Recorded } & \multicolumn{2}{c}{$\begin{array}{c}\text { Not } \\
\text { recorded }\end{array}$} & Done & \multicolumn{2}{c}{$\begin{array}{c}\text { Not } \\
\text { done }\end{array}$} \\
\cline { 2 - 9 } & No. & $\%$ & No. & $\%$ & No. & $\%$ & No. & $\%$ \\
\hline Blood gases & 32 & 53.3 & 28 & 45.7 & 32 & 53.3 & 28 & 45.7 \\
Chest X-ray & 0 & 0.0 & 60 & 100.0 & 58 & 96.7 & 2 & 3.3 \\
Pulse oximeter & 0 & 0.0 & 60 & 100.0 & 60 & 100.0 & 0 & 0.0 \\
Continuous ECG & 0 & 0.0 & 60 & 100.0 & 0 & 0.0 & 60 & 100.0 \\
\hline
\end{tabular}

Table (7): Weaning from CPAP.

\begin{tabular}{lcccr}
\hline & \multicolumn{2}{c}{ Recorded } & \multicolumn{2}{c}{ Not recorded } \\
\hline & No. & $\%$ & No. & $\%$ \\
\hline Weaning & 60 & 100 & 0 & 0.0 \\
Gradually \& successful & 35 & 58.3 & 0 & 0.0 \\
Failed & 25 & 41.7 & 0 & 0.0 \\
\hline
\end{tabular}

Table (8): Outcome.

\begin{tabular}{lcccc}
\hline & \multicolumn{2}{c}{ Recorded } & \multicolumn{2}{c}{ Not recorded } \\
\cline { 2 - 5 } & No. & $\%$ & No. & $\%$ \\
\hline Improved & 35 & 58.3 & 0 & 0.0 \\
Failed: & 25 & 41.7 & 0 & 0.0 \\
1- Central apnea (significant) & 12 & 48.0 & & \\
2- Haemorragic disease & 2 & 8.0 & & \\
3- Sepsis & 6 & 24.0 & & \\
4- Congenital anomalies & 5 & 20.0 & & \\
\hline
\end{tabular}




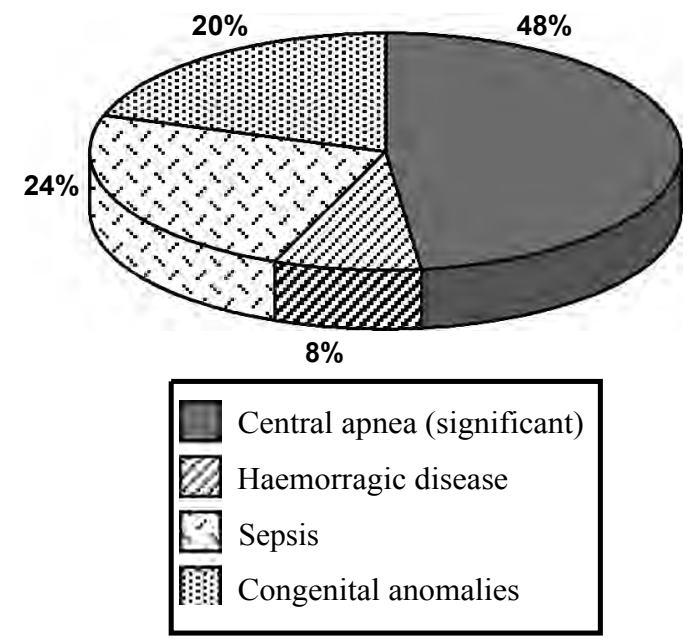

Fig. (1): Causes of CPAP failure.

\section{Discussion}

Our study included 60 neonates receiving BCPAP care as it is the only available type of CPAP in our NICU. Our study is supported by Tagare et al., 2013 [3] study that was comparing the use of Bubble CPAP and ventilator CPAP (conventional CPAP). Their results showed that Bubble CPAP has higher success rate than ventilator CPAP $(82.5 \%$ vs. $63.2 \%)$.

In our study only 12 cases $(20 \%)$ were given surfactant before CPAP use. However CPAP and surfactant work together towards establishing and maintaining functional residual capacity in RDS. Kandraju et al., 2013 [4] stated that the need for mechanical ventilation was significantly less in early rescue surfactant group (16.2\% vs. $31.6 \%)$.

In our study weaning from CPAP was gradual and successful in $58.3 \%$ of cases by using the guidelines. In our NICU newborns were kept on NCPAP until they were on $\mathrm{FiO}_{2}=0.21$ and pressure of $5 \mathrm{cmH}_{2} \mathrm{O}$ and then were weaned off NCPAP completely. Failure occurred in $41.7 \%$ of cases and this failure of weaning was due to failure in CPAP system in those cases which results in sudden disconnection of CPAP to connect the patient to mechanical ventilation or death. Other studies supporting our technique of weaning such as Todd et al., 2012 [5] study stated that weaning should be done when neonates were clinically stable on CPAP $4-6 \mathrm{~cm}$ with $\mathrm{FiO}_{2}<25 \%$ for at least 12 hours and that method one (Taken 'OFF' CPAP with the view to stay 'OFF' completely) significantly shortened CPAP weaning time, CPAP duration, oxygen duration, BPD and length of admission.

In our study the outcome of BCPAP showed improvement in $58.3 \%$ of cases, failure here means worsening of medical conditions of the cases that ended by connection to mechanical ventilation or death. Causes of failure were; frequent central apnea in $12(48 \%)$ cases, hemorrhagic disease of the newborn in $2(8 \%)$ cases, severe sepsis in 6 (24\%) cases and congenital anomalies (mainly cardiac) in $5(20 \%)$ cases. Our results were in agreement with that represented by Maiya et al., 2009 [6] who stated that failure was in $20 \%$ they related that to severe RDS (down score 7 at admission ), gestational age (28wks or less), low birth weight especially from $750 \mathrm{gm}$ to $1250 \mathrm{gm}$, antenatal steroids (no exposure or partial exposure increase risk).

\section{Conclusion:}

Data of the study showed that using NCPAP for cases of respiratory distress in neonatal Intensive Care Unit in Assiut University Children Hospital partially follow the reference standard guidelines of the study.

\section{Recommendations:}

- Following the guidelines about indications, contraindications and investigations of the use of CPAP as it is partially followed by resident doctors in NICU in AUCH.

- Regular refreshment of resident doctors information about guidelines for using of CPAP as many of them don't know other indications of CPAP rather than RDS.

- Don't use CPAP when it is not indicated as it will fail.

- Recording all data in patient sheet as there is big defect in recording data especially data of CPAP set up and monitoring in the Neonatal Intensive Care Unit in Assiut University Children Hospital.

- Considering addition of master sheet containing data about CPAP set up and monitoring to patient sheet to insure recording of all these data and not to be missed.

- Clinical monitoring and regular check of CPAP system during use must be done every 2-4 hours as recommended by guidelines to insure good results and early detection of complications.

- Investigations should be done when needed with no over use of investigations as doing blood gases several times without obvious causes and also doing X-ray for almost all patients without obvious cause.

- Do not use mechanical ventilation while cases can benefit from use of CPAP unless it is indicated. 
- Periodic examination of nasal septum to detect early any necrosis of the septum.

- Insure availability of variable sized nasal prongs that can fit any neonate to minimize the risk of nasal trauma.

\section{Acknowledgements:}

The authors express their deep sense of gratitude to all medical staff members and all workers in NICU of AUCH for their encouragement and kind endless help.

\section{Limitations of study:}

- Deficiency of recording data in patient sheet.

- Small numbers of newborns receiving CPAP care.

No conflicts of interest.

\section{References}

1- KIRAN S., MURKI S., PRATAP O.T., KANDRAJU H. and REDDY A.: Nasal continuous positive airway pressure therapy in a non tertiary Neonatal Unit: Reduced need for up-transfers. Indian J. Pediatr., Jun. 21; 50: 905-15, 2014.

2- KOTI, MURKI S., GADDAM P., REDDY A. and REDDY M.D.R.: Bubble CPAP for respiratory distress syndrome in preterm infants. Indian Pediatr., 47, 2010.

3- TAGARE, KADAM S., VAIDYA U., PANDIT A. and PATOLE S.: Bubble CPAP versus ventilator CPAP in preterm neonates with early onset respiratory distressrandomized controlled trial. J. Trop. Pediatr., 59: 113-9, 2013.

4- KANDRAJU H., MURKI S., SUBRAMANIAN S., GADDAM P., DEORARI A. and KUMAR P.: Early routine versus late selective surfactant in preterm neonates with respiratory distress syndrome on nasal continuous positive airway pressure: A randomized controlled trial. Neonatology, 103 : 148-54, 2013.

5- TODD D.A., WRIGHT A., BROOM M., CHAUHAN M., MESKELL S., CAMERON C., et al.: Methods of weaning preterm babies < 30 weeks gestation off CPAP: A multicentre randomised controlled trial. Arch. Dis. Child. Fetal. Neonatal. Ed., 97: F236-40, 2012.

6- MAIYA P.P., URS P.S. and KHAN F.: Bubble CPAP-a primary respiratory support for respiratory distress syndrome in newborns. Indian Pediatr., 46 (5): 409-11, 2009.

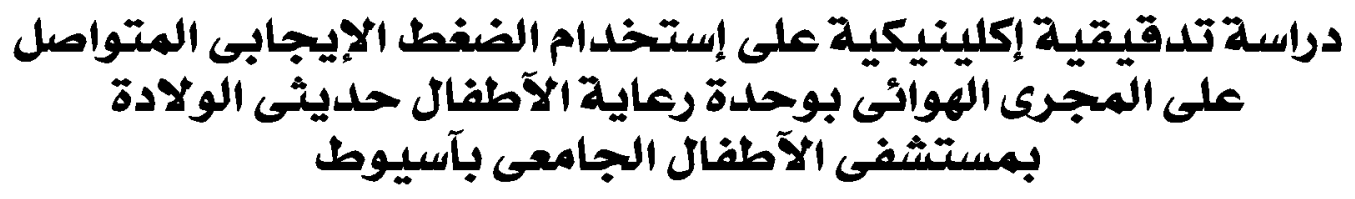

الضغط الإيجابى المتواصل على المجرى الهوائى هو إستخدام ضغط إيجابى على الممرات الهوائية لطفل يتنفس تلقائيا خلال دورته التنفسية

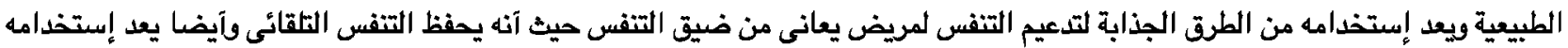
آقل ضررا على الجهاز التنفى حيث آن نسبة حدوث آمراض رئوية مزمنة نتيجة لإستخدامه آقل مما يحدث نتيجة لإستخدام آجهزة التنفس الصناعي.

آهداف البحث: تقيم إلى آى حد يتم تطبيق النظم المتبعة فى إستخدام الضغط الإيجابى المتواصل على المجرى الهوائى بوحدة رعاية

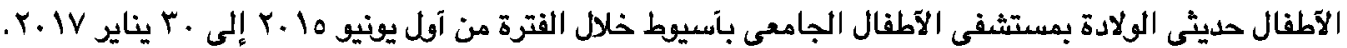

الفئات المستهدفة: كل الآطفال الذين تلقوا رعاية بوحدة الآطفال حديثى الولادة بمستشفى الآطفال الجامعى بآسيوط بإستخدام الضغط

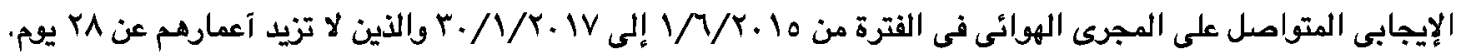

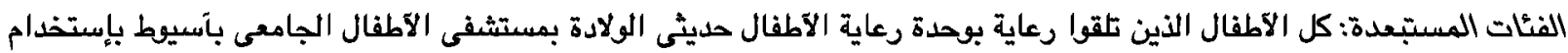

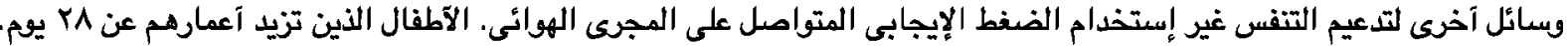

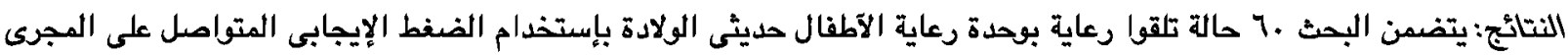

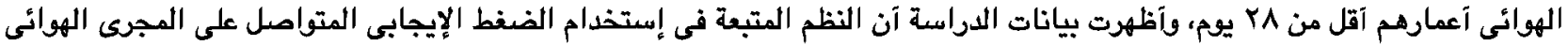
آتبعت جزنيا المعايير المرجعية اللدراسة منئ. 